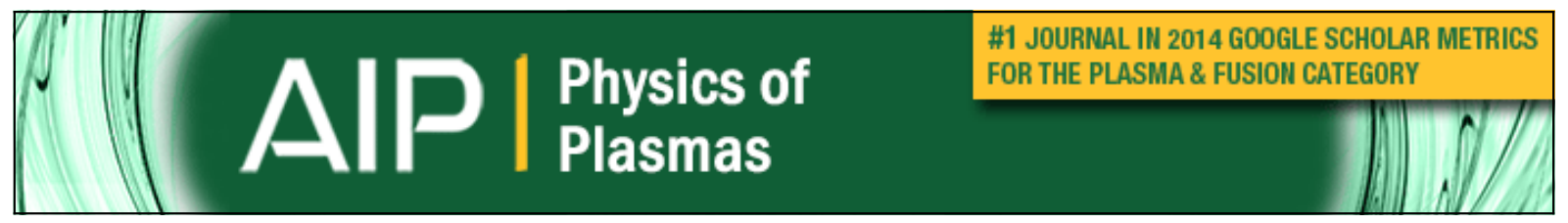

\title{
Excitation of the $\mathrm{I}=\mathbf{2}$ diocotron mode with a resistive load
}

G. Bettega, F. Cavaliere, B. Paroli, R. Pozzoli, M. Romé, and M. Cavenago

Citation: Physics of Plasmas 15, 032102 (2008); doi: 10.1063/1.2890773

View online: http://dx.doi.org/10.1063/1.2890773

View Table of Contents: http://scitation.aip.org/content/aip/journal/pop/15/3?ver=pdfcov

Published by the AIP Publishing

\section{Articles you may be interested in}

Trapped-particle diocotron modes

Phys. Plasmas 10, 3492 (2003); 10.1063/1.1599356

Finite length diocotron modes

Phys. Plasmas 8, 407 (2001); 10.1063/1.1340856

Subharmonic autoresonance of the diocotron mode

Phys. Plasmas 7, 1712 (2000); 10.1063/1.873989

Destabilization of diocotron modes inside structured anode cavities

Phys. Plasmas 6, 323 (1999); 10.1063/1.873711

The finite length diocotron mode

Phys. Plasmas 5, 601 (1998); 10.1063/1.872752

\section{Did your publisher get}

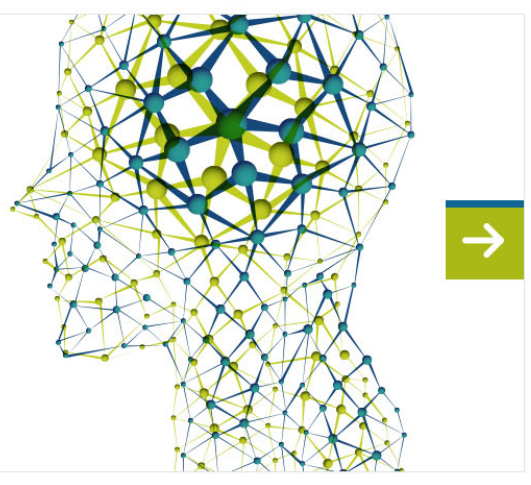




\title{
Excitation of the $I=2$ diocotron mode with a resistive load
}

\author{
G. Bettega, ${ }^{1}$ F. Cavaliere, ${ }^{1}$ B. Parolii, ${ }^{1}$ R. Pozzoli,${ }^{1}$ M. Romé, ${ }^{1}$ and M. Cavenago ${ }^{2}$ \\ ${ }^{1}$ I.N.F.N. Sezione di Milano and Dipartimento di Fisica, Università degli Studi di Milano, \\ Via Celoria 16, Milano I-20133, Italy \\ ${ }^{2}$ I.N.F.N. Laboratori Nazionali di Legnaro, Viale dell'Università 2, I-35020 Legnaro, Italy
}

(Received 28 December 2007; accepted 13 February 2008; published online 14 March 2008)

\begin{abstract}
The resistive wall instability of the $l=2$ diocotron mode in a pure electron plasma has been investigated with a systematic variation of the parameters of the external impedance connected to a pair of sectored electrodes. The measured growth rate is well described by a linear perturbation theory of the two-dimensional drift-Poisson system. (C) 2008 American Institute of Physics.
\end{abstract}

[DOI: $10.1063 / 1.2890773$ ]

\section{INTRODUCTION}

Theoretical and experimental studies on single charged (or partially neutralized) plasmas play an important role in the fields of basic plasma physics (equilibria, instabilities, waves propagation), atomic physics (antimatter production and storage), and fluid dynamics [two-dimensional (2D) vortex dynamics and turbulence, self-organization].

Pure electron plasmas can be stored in cylindrical Malmberg-Penning traps ${ }^{1}$ for very long times (up to minutes) with external static electric and magnetic fields. The base configuration of the device consists (see Fig. 1) of three cylindrical electrodes, the inner being grounded, while the outer are kept at a fixed potential (typically tens or hundreds of volts) in the presence of a constant and uniform magnetic field $B$ directed along the axis of the device. High vacuum conditions $\left(p \leqslant 10^{-8}\right.$ mbar) are required in order to reduce electron-neutral collisions which determine the radial expansion of the plasma. Interparticle (electron-electron) collisions drive the plasma toward a thermal equilibrium state in a few hundreds of milliseconds. The thermal equilibrium state is characterized by an almost flat density profile, and by a shear-free bulk rotation of the trapped column.

The main diagnostics are optical and electrostatic. In the former case, the plasma density distribution at any time can be obtained dumping the trapped charge onto a phosphor screen kept at high voltage $(10-15 \mathrm{kV})$, and collecting the emitted light by a triggered CCD (charge coupled device) camera. The evolution of the axially averaged plasma density distribution can be reconstructed with a sequence of plasma snapshots, obtained with an increasing trapping time (the "jitter" in the initial conditions is less than $0.01 \%$ ). The electrostatic diagnostic is conversely passive: The plasma electrostatic oscillations are followed in time analyzing the induced current signals, which form on the confinement cylindrical electrodes used as receiving antennas. Azimuthally sectored cylinders allow us to diagnose plasma modes with an angular dependence $\exp (i l \theta), l$ being an integer. The frequencies and amplitudes of the modes can be related to the main plasma physical parameters, i.e., density and temperature. ${ }^{2}$

In Ref. 3 , it was shown that the pure $l=1$ diocotron mode is destabilized when a wall sector is connected to an external
$R C$ circuit. The analysis has been performed measuring the growth rate of the mode. Here the attention is focused on the effect of an external resistive load on the evolution of the $l$ $=2$ mode, which consists in an elliptical deformation of the plasma cross section (quadrupole perturbation). ${ }^{4,5}$ In Ref. 6 , it was found that the linear decay rate of the $l=2$ mode is reduced when an external resistive load is applied, thus showing that the mode has negative energy. ${ }^{7}$ The $l=2$ mode is excited in the plasma by an octupole electrode and diagnosed by means of the charge induced on a sector of a second octupole electrode. The excited mode exhibits a linear decay that is reduced when an external resistive load is applied.

The experiments reported here are based on a direct electrostatic measurement of the linear growth rate of the perturbation, following an approach similar to that of Ref. 3 . The mode is initially present in the plasma with a very low amplitude. Its evolution is measured by means of an almost nonperturbative detection of the induced current. When a resistive load is applied, a finite growth rate is measured that agrees with the estimate of the linear analysis.

The paper is organized as follows. In Sec. II, some parameters of the experimental apparatus are given, and the $2 \mathrm{D}$ approximation adopted for the description of the trapped plasma evolution is briefly introduced. The diagnostic techniques are described, and the experimental results on the destabilization of the mode due to an external resistive load are presented. A 2D cold fluid model, which takes into account the coupling between plasma modes and the external resistive network, is developed in Sec. III, and a comparison between the theoretical predictions and the experimental results is presented. Section IV is devoted to short conclusions.

\section{EXPERIMENTAL APPARATUS AND EXCITATION OF THE MODE}

The experiments have been performed in the MalmbergPenning trap ELTRAP. ${ }^{8}$ The device has eight cylindrical electrodes with length $L=9 \mathrm{~cm}$ and two azimuthally sectored electrodes, with length $L_{S}=15 \mathrm{~cm}$, made of two and four angular patches, respectively. The internal radius of the conducting cylinders is $R_{W}=4.5 \mathrm{~cm}$. A plasma column with a length $L_{P}=20-80 \mathrm{~cm}$ and a radius $R_{P}=1.0-2.0 \mathrm{~cm}$ can be 


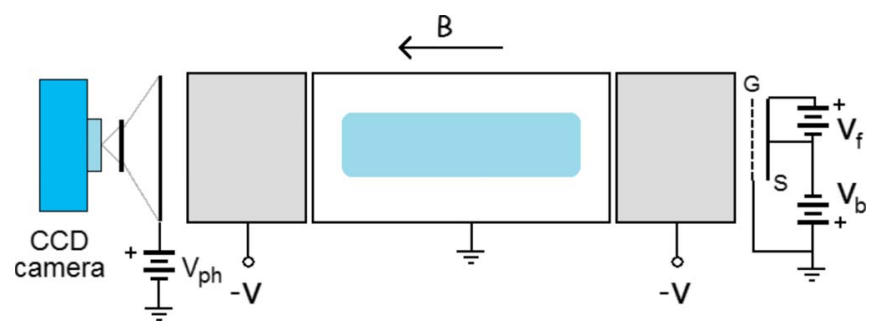

FIG. 1. (Color online) Schematic of the base configuration of a cylindrical Malmberg-Penning trap. The electron plasma is confined with $|V|$ $=50-100 \mathrm{~V}$ and $B=0.05-0.15 \mathrm{~T}$. The phosphor screen is positively biased at $V_{\mathrm{ph}}=10-15 \mathrm{kV}$. The CCD camera is activated by an external signal synchronized with the dump of the plasma. The electron source (S) is a thermionic cathode negatively biased at a voltage $V_{b}$ with respect to a grounded grid $(\mathrm{G})$.

trapped, using a magnetic field with strength up to $B$ $=0.2 \mathrm{~T}$. The plasma is generated by a spirally shaped thermionic cathode, kept at a negative potential with respect to a grounded grid. The electrons are injected into the system when the electrode from the source side is shortly grounded.

Typical plasma parameters are as follows: Electron density $n=10^{6}-10^{7} \mathrm{~cm}^{-3}$, temperature $T=5-10 \mathrm{eV}$, gyroradius $\rho_{L}=100-500 \mu \mathrm{m}$, and Debye length $\lambda_{D}=0.1-0.5 \mathrm{~cm}$. The characteristic time scales are defined by the cyclotron frequency $\nu_{c} \approx 1 \mathrm{GHz}$, the plasma frequency $\nu_{p} \approx 20-80 \mathrm{MHz}$, the axial bounce frequency $\nu_{b} \approx 1-5 \mathrm{MHz}$, and the equilibrium rotation frequency $\omega_{D} / 2 \pi=\nu_{D} \approx 50-500 \mathrm{kHz}$, where $\omega_{D}=e n / 2 \epsilon_{0} B$.

The characteristic lengths and frequencies, therefore, satisfy the following inequalities: $\rho_{L} \ll \lambda_{D} \ll R_{P} \ll L_{P}, \nu_{c} \gg \nu_{p}$ $\gg \nu_{b}>\nu_{D}$. On the base of these scalings, the electron plasma dynamics is well approximated by the system of 2D cold fluid equations (drift-Poisson system), $\partial n / \partial t+\mathbf{v} \cdot \nabla n=0, \mathbf{v}$ $=-\nabla \phi \times \mathbf{B} /|\mathbf{B}|^{2}, \nabla^{2} \phi=e n / \epsilon_{0}$, for the particle density $n$, the velocity field $\mathbf{v}$, and the electrostatic potential $\phi$.

In previous works, the $l=2$ mode was excited by means of a tuned resonant drive ${ }^{4}$ or a voltage pulse, ${ }^{5}$ and the subsequent mode damping and nonlinear oscillations were studied with the optical diagnostic, measuring the elliptical deformation of the plasma cross section. In the experiments described here, the evolution of the $l=2$ diocotron mode has been measured with the electrostatic diagnostic, using two electrically connected opposite $\pi / 2$ patches of the four sectored cylinder. Such an antenna configuration has sensitivity toward quadrupole perturbations, and no sensitivity toward odd order diocotron modes (in particular toward the $l=1$ diocotron mode).

The experimental setup is shown in Fig. 2. Two opposite azimuthal sectors are connected to a computer controlled switch. In the rest state of the switch the antennas are grounded, while in the active state they are connected to an adjustable resistance $R$. Within the frequency range of interest (of the order of $\nu_{D}$ ), the effect of the transmission line (internal high vacuum cables and external coaxial cables) can be modeled by a fixed capacitance $C$, which takes into account also the small capacitance of the antennas with respect to the ground. The effect of the total system capacitance on the evolution of the mode can be studied adding an external

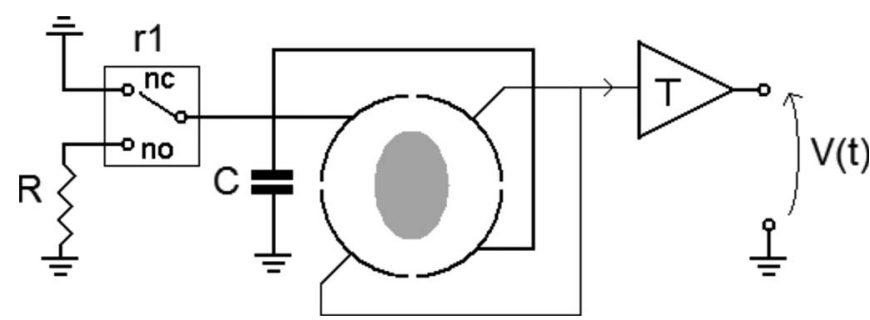

FIG. 2. Schematic of the electrical connections for the excitation and the electrostatic diagnostic of the $l=2$ mode. $\mathbf{r} 1$ is a computer controlled switch. The resistance $R$ can be arbitrarily varied; $C$ is the total system capacitance (approximately that of the coaxial cables connecting the electrodes to the external circuits). A transimpedance amplifier is used to read the induced charge at the wall.

capacitor. The other two electrodes (electrically connected) work as receiving antennas and are used for diagnostic purposes. The time variation of the induced charge on the antennas is directly read by a transimpedance amplifier, which outputs a voltage signal $V(t)$ proportional to the induced current. ${ }^{9}$ This kind of diagnostic is (almost) nonperturbative, and its main features will be described in detail elsewhere. The output signal is continuously sampled at $f_{S}=500 \mathrm{kHz}$ with a time window of 4096 points, for a frequency resolution of $\Delta f=122 \mathrm{~Hz} ; 16$ bit amplitude sampling is available, for a voltage resolution of $\Delta V=190 \mu \mathrm{V}$.

The $l=2$ mode determines a clearly distinguishable peak in the Fourier spectrum of the received signal $V(t)$ whose amplitude and frequency shifts are followed by a software peak detector, which works as long as the signal is well above the noise level. The mode is identified through the ratio between its frequency and the frequency of the $l=1$ diocotron mode, which is naturally present and can be separately measured using a dipole antenna (a single patch of the $\pi$-sectored electrode). ${ }^{10}$ According to the linear theory for the diocotron modes, ${ }^{11}$ the $l$-th mode frequency is $\omega^{(l)}$ $=\omega_{D}\left[l-1+\left(R_{P} / R_{W}\right)^{2 l}\right]$, so that $\omega^{(2)} / \omega^{(1)} \approx\left(R_{W} / R_{P}\right)^{2} \approx 12.6$ is expected (the plasma radius $R_{P}$ is separately measured with the optical diagnostic, see Sec. I). Referring to Fig. 3, it is experimentally found that $\omega^{(1)} / 2 \pi=7.5 \mathrm{kHz}$, and $\omega^{(2)} / 2 \pi$ $=95.6 \mathrm{kHz}$ (these frequency values give confidence in the nature of the revealed peak). Note that during the experiment the $l=1$ and 2 modes evolve independently. Their evolutions
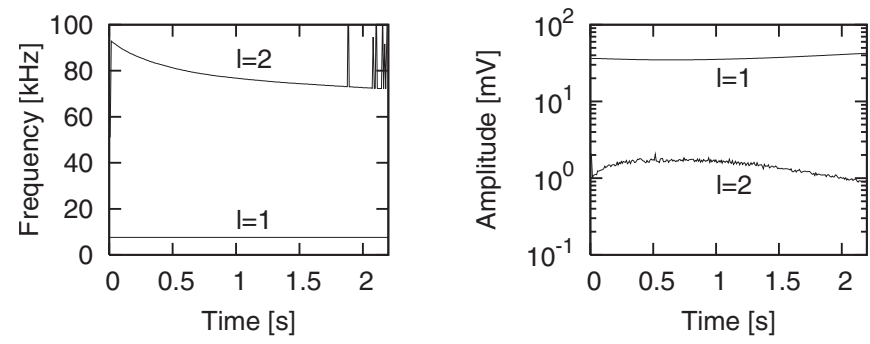

FIG. 3. Time evolutions of frequency (left) and amplitude (right) of the $l$ $=1$ and $l=2$ diocotron modes. The $l=1$ mode is measured separately with a $\pi$-sectored probe. During the natural evolution of the $l=2$ mode, the $l=1$ keeps a nearly constant amplitude. The plasma parameters are as follows: Plasma length $L_{P} \simeq 50 \mathrm{~cm}$, density $n \simeq 5.1 \times 10^{12} \mathrm{~m}^{-3}$, and magnetic field $B=0.11 \mathrm{~T}$. 


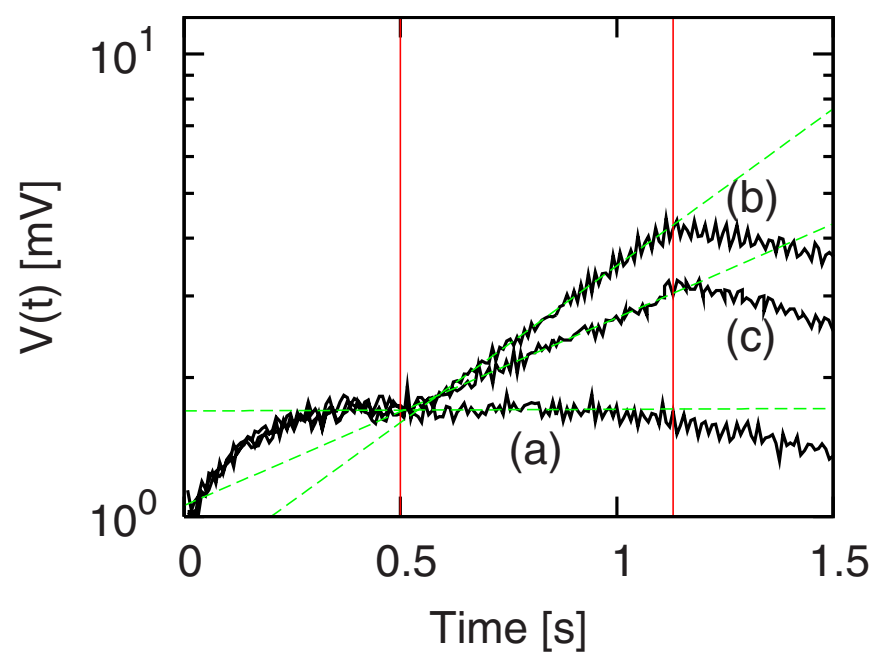

FIG. 4. (Color online) Effect of a resistive load on the time behavior of the $l=2$ diocotron mode, monitored through the voltage signal $V(t)$. (a) $R$ $=0 \Omega, \quad \gamma^{(2)} \simeq 8.23 \times 10^{-3} \mathrm{~s}^{-1}$; (b) $R=2 \mathrm{k} \Omega, \quad \gamma^{(2)} \simeq 1.56 \mathrm{~s}^{-1}$; (c) $R=8 \mathrm{k} \Omega$, $\gamma^{(2)} \simeq 9.34 \times 10^{-1} \mathrm{~s}^{-1}$. The resistance is connected to the antennas at $t$ $=0.5 \mathrm{~s}$ for a time interval $\Delta t=0.63 \mathrm{~s}$. The total line capacitance is $C$ $\simeq 900 \mathrm{pF}, L_{P} \simeq 50 \mathrm{~cm}$, and $B=0.11 \mathrm{~T}$.

up to $2.2 \mathrm{~s}$ are shown in Fig. 3. The $l=1$ mode has actually been measured during a longer time (up to $16 \mathrm{~s}$ ). A slow exponential growth has been found, with $\gamma^{(1)} \approx 0.097 \mathrm{~s}^{-1}$, and a final $\delta \omega^{(1)} / \omega^{(1)} \approx 0.1$ increase in the frequency. The natural evolution of the $l=2$ is instead characterized by an initial amplitude increase and a nearly stable or slowly decreasing behavior, while its frequency monotonically decreases during the whole evolution. At $t=2.2 \mathrm{~s}$, the $l=2$ mode is fully masked by the noise. The two oscillations are well separated in frequency, and the $l=1$ amplitude remains nearly constant during the whole evolution of the $l=2$ mode, so that no mutual interaction is expected to occur during the measurements.

\section{A. Excitation of the $I=2$ mode with a resistive load}

The effects of the resistive load on the evolution of the $l=2$ mode are clearly visible in the received voltage signal $V(t)$; see Fig. 4. After the injection the plasma freely evolves for $0.5 \mathrm{~s}$, then the switch is turned on up to $t=1.63 \mathrm{~s}$, after that the antennas are grounded again up to the end of the cycle. Data acquisition stops when the $l=2$ peak reaches the noise level (this is indicated by the large fluctuations of the measured mode frequency, due to the failure of the software peak detector; see Fig. 3, left). When the resistive load is connected to the antennas, the $l=2$ mode is destabilized and grows exponentially in time.

\section{LINEAR ANALYSIS}

The resistive wall destabilization of the $l=1$ diocotron mode was originally estimated quantitatively using the balance between the power of the mode, calculated as the time variation of the electrostatic interaction energy between the plasma and its image charge, and the power thermally dissipated by the induced current in the external resistor. ${ }^{3}$ For a generic $l$, the growth rate of the perturbation may be esti- mated by means of a first-order analysis of the 2D driftPoisson system. The coupling between the plasma dynamics and the external circuit can be taken into account by the introduction of a time-dependent boundary condition on the wall of the confining cylinder. Although the antenna has a finite length, the use of a 2D model is justified by the consideration that externally imposed electric signals (or coming, as in the present case, from the response of a passive network stimulated by the induced charge) at a frequency of the order of a few $\omega_{D}$ induce a pure 2D plasma response, because the modes with a nonzero axial component (Trivelpiece-Gould modes ${ }^{12}$ ) have a much higher frequency than $\omega_{D}$.

The perturbed density and electrostatic potential are written as time and space Fourier series $n(r, \theta, t)=n_{0}(r)$ $+\sum_{l=-\infty}^{\infty} \delta n^{(l)}(r) \exp \left[i\left(l \theta-\omega^{(l)} t\right)\right] \quad$ and $\quad \phi(r, \theta, t)=\phi_{0}(r)$ $+\sum_{l=-\infty}^{\infty} \delta \phi^{(l)}(r) \exp \left[i\left(l \theta-\omega^{(l)} t\right)\right]$, where $n_{0}(r)$ and $\phi_{0}(r)$ represent the equilibrium density and electrostatic potential, respectively. In the previous relations, $\omega^{(l)}$ is the complex frequency of the $l$-th Fourier mode. The assumption on the time dependence of the perturbations is justified by the fact that the response of the plasma is studied in a stationary regime, and, as it is experimentally verified, $1 / \gamma^{(2)} \gg\left[2 \pi / \omega^{(2)}\right]$, i.e., the amplitude of the mode grows very slowly while plasma and external circuit are coupled.

The equation for the density perturbation is

$$
\delta n^{(l)}(r)=\frac{-(l / r B) \delta \phi^{(l)}(r) d n_{0}(r) / d r}{\omega^{(l)}-l \omega_{r e}(r)},
$$

where $\omega_{r e}(r)=(1 / r B)\left(d \phi_{0}(r) / d r\right)$ is the equilibrium angular velocity profile. Combining Eq. (1) with the linearized Poisson equation, and considering a flat density equilibrium profile $n_{0}(r)=n_{0}\left[1-H\left(r-R_{P}\right)\right]$ with radius $R_{P}$ (where $H$ represents Heaviside's step function), the following equation for the perturbation of the electrostatic potential is obtained:

$$
\begin{aligned}
\frac{1}{r} & \frac{d}{d r} r \frac{d}{d r} \delta \phi^{(l)}(r)-\frac{l^{2}}{r^{2}} \delta \phi^{(l)}(r) \\
& =\frac{2 l \omega_{D}}{\omega^{(l)}-l \omega_{D}} \frac{\delta \phi^{(l)}(r)}{r} \delta\left(r-R_{P}\right) .
\end{aligned}
$$

Equation (2) is solved in the intervals (I) $0 \leqslant r<R_{P}$ and (II) $R_{P}<r \leqslant R_{W}$. The two solutions are matched in $r=R_{P}$, and an additional condition relating them is obtained by applying the operator $\lim _{\epsilon \rightarrow 0^{+}} \int_{R_{P}-\epsilon}^{R_{P}+\epsilon} d r r$ to Eq. (2). A unique solution is found by imposing the boundary condition at $r=R_{W}$, which takes into account the effect of the coupling between the induced charge oscillations at the antennas and the external network. To compute the value of the potential on the confining wall at a given instant, the current stimulus has to be estimated first. The induced charge on the wall is

$$
\begin{aligned}
\delta Q(t)= & -\left.\epsilon_{0} L_{S} R_{W} \sum_{l=-\infty}^{\infty} \frac{d \delta \phi^{(l)}(r)}{d r}\right|_{r=R_{W}} \exp \left(-i \omega^{(l)} t\right) \\
& \times \int_{\text {probes }} d \theta \exp (i l \theta) .
\end{aligned}
$$

The integral over the azimuthal angle $\theta$ depends on the ge- 
ometry of the antennas. In particular, in the present case it is $4 i / l$ for $l=4 k+2$ (with $k$ a relative integer), and zero otherwise. Under stationary conditions, the induced current is $i(t)=\sum_{l=-\infty}^{\infty} l^{(l)} \exp \left(-i \omega^{(t)} t\right)$, where the complex amplitudes are given by

$$
I^{(l)}=-\left.\left(\frac{4 \epsilon_{0} L_{S} R_{W}}{l}\right) \omega^{(l)} \frac{d \delta \phi^{(l)}(r)}{d r}\right|_{r=R_{W}} .
$$

The plasma is electrically equivalent to a series of sinusoidal current generators with complex amplitude $I^{(l)}$ and connected in parallel. The $l$-th voltage amplitude on the antennas is $V^{(l)}=Z\left(\omega^{(l)}\right) I^{(l)}$, where $Z(\omega)=R(1-i \omega R C) /\left(1+\omega^{2} R^{2} C^{2}\right)$ represents the impedance of the external circuit. The timedependent electrostatic potential at the wall is then

$$
\begin{aligned}
\delta \phi^{(l)}\left(r=R_{W}\right)= & -\left(\frac{8 \epsilon_{0} L_{S} R_{W}}{i \pi l^{2} C}\right) \\
& \times\left.\frac{\omega^{(l)} R C\left(1-i \omega^{(l)} R C\right)}{1+\omega^{(l) 2} R^{2} C^{2}} \frac{d \delta \phi^{(l)}(r)}{d r}\right|_{r=R_{W}} .
\end{aligned}
$$

The dispersion relation then reads

$$
\omega^{(l)}=\omega_{D}\left[l-1+\left(\frac{R_{P}}{R_{W}}\right)^{2 l} \frac{1+k^{(l)}}{1-k^{(l)}}\right],
$$

where

$$
k^{(l)} \equiv i \epsilon^{(l)} \frac{\omega^{(l)} R C\left(1-i \omega^{(l)} R C\right)}{1+\omega^{(l) 2} R^{2} C^{2}}
$$

and $\epsilon^{(l)}=\left(8 \epsilon_{0} L_{S}\right) /(\pi l C)$.

The frequency of the $l$-th azimuthal mode is shifted with respect to the case of the constant (zero) voltage boundary condition by $\delta \omega^{(l)} \simeq 2 \omega_{D}\left(R_{P} / R_{W}\right)^{2 l}\left(L_{S} / L_{P}\right) k^{(l)}$, where the approximation $\left(1+k^{(l)}\right) /\left(1-k^{(l)}\right) \approx 1+2 k^{(l)}$ has been used (this approximation is very well justified using the typical experimental parameters) and the finite length of the plasma and of the wall sector have been taken into account. This can be justified in terms of power balance, as discussed in Ref. 3 .

The real part of the frequency shift is

$$
\alpha^{(l)}=\operatorname{Re}\left[\delta \omega^{(l)}\right]=\frac{16 \omega_{D} \epsilon_{0} L_{S}^{2}}{\pi l C L_{P}}\left(\frac{R_{P}}{R_{W}}\right)^{2 l} \frac{\omega^{(l) 2} R^{2} C^{2}}{1+\omega^{(l) 2} R^{2} C^{2}},
$$

and it has the meaning of a frequency slip, whereas the imaginary part

$$
\gamma^{(l)}=\operatorname{Im}\left[\delta \omega^{(l)}\right]=\frac{16 \omega_{D} \epsilon_{0} L_{S}^{2}}{\pi l C L_{P}}\left(\frac{R_{P}}{R_{W}}\right)^{2 l} \frac{\omega^{(l)} R C}{1+\omega^{(l) 2} R^{2} C^{2}}>0
$$

gives the (exponential) growth rate of the perturbation, which follows the behavior of the dissipated power proportionally to the real part of the impedance of the $R C$ circuit.

The theoretical results have been compared with a set of data obtained by varying the resistive load $R$ in the range $0-100 \mathrm{k} \Omega$, for different values of the total capacitance $C$. The mode growth rate as a function of $R$, for three different values of the external capacitance, is shown in Fig. 5. The comparison with the linear theory prediction has been performed using Eq. (9) for the case $l=2$, and the experimen-

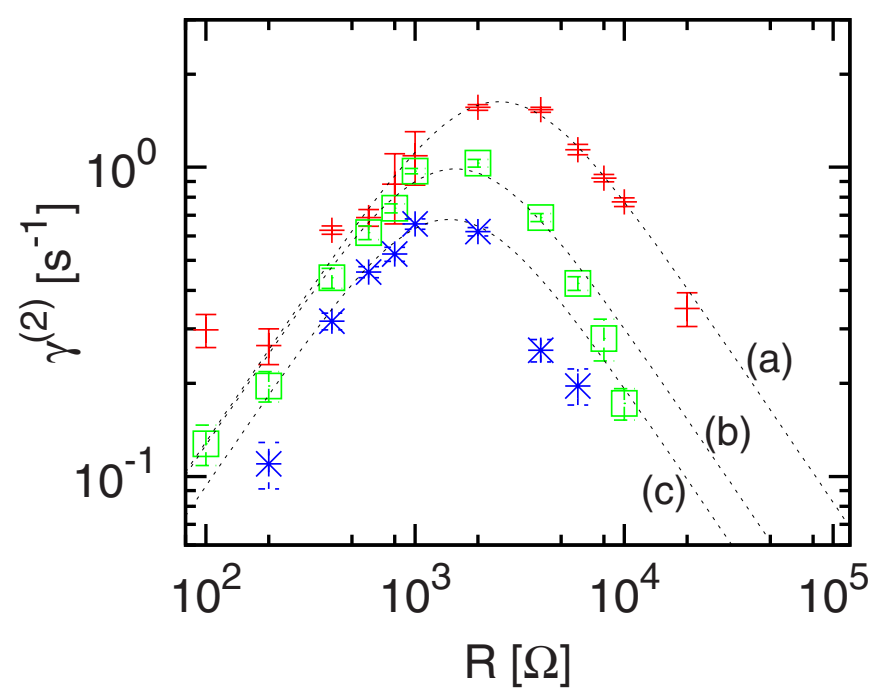

FIG. 5. (Color online) Measured growth rates $\gamma^{(2)}$ (points) as a function of the resistive load $R$, plotted for three values of the total line capacitance $C$ and compared with the analytical result, Eq. (9) (dotted lines). Each $\gamma^{(2)}$ value is obtained averaging on $\geqslant 30$ machine cycles. The data refer to (a) red ,$+ n \simeq 5.32 \times 10^{12} \mathrm{~m}^{-3}$ and $C \simeq 900 \mathrm{pF}$; (b) green $\square, n \simeq 5.27 \times 10^{12} \mathrm{~m}^{-3}$ and $C \simeq 1.37 \mathrm{nF}$; (c) blue $*, n \simeq 4.52 \times 10^{12} \mathrm{~m}^{-3}$ and $C \simeq 1.84 \mathrm{nF}$. The other experimental parameters are the same as in Fig. 4.

tally determined (in an independent way) plasma density value $n$. The value of $L_{P}$ has been estimated as the length of the trapping region (the distance between the axially confining electrodes). The agreement between theory and experiment is good for intermediate values of the resistance (around the maximum of the resistive growth rate), while deviations are found at low and high $R$ values, for all values of $C$. In these ranges, the very small detected values of $\gamma$ are more strongly affected by noise, which is mainly determined, after the signal conditioning, by the electronics of the data acquisition board. The presence of a non-negligible natural damping rate of the mode (which is absent in the theoretical treatment described above due to the choice of the plasma equilibrium profile) can also affect the results. The damping rate measured after switching off the resistive excitation of the mode turns out to be of the order of a few $0.1 \mathrm{~Hz}$ (consistent with the natural damping rate of the $l=2$ mode measured in previous experiments ${ }^{5}$ ).

Note that no significant real frequency shift is detected in the experiments, since the maximum real frequency shift $\alpha^{(2)}$ expected from Eq. (8) is below $0.1 \mathrm{kHz}$, and is lower than the frequency resolution $\Delta f$, which can be achieved with the experimental method.

\section{CONCLUSIONS}

The resistive wall destabilization of the $l=2$ diocotron mode has been investigated measuring the growth rate by means of an almost nonperturbative electrostatic diagnostic. The experimental results are well described in their main features by the linear perturbative analysis of the 2D driftPoisson equations.

The theory predicts an exponential behavior of the $l=2$ diocotron mode when dissipation is included in the system, 
in agreement with the nature of the diocotron modes as negative energy waves. In particular, the measured growth rate of the mode versus the external resistance $R$ follows the theoretical prediction, showing the calculated dependence on the total system capacitance. Deviations are found that may be related to a non-step-like equilibrium density profile of the plasma and to finite length (non-2D) effects ${ }^{13}$ not included in the model.

${ }^{1}$ J. H. Malmberg and J. S. deGrassie, Phys. Rev. Lett. 35, 577 (1975).

${ }^{2}$ N. Shiga, F. Anderegg, D. H. E. Dubin, C. F. Driscoll, and R. W. Gould, Phys. Plasmas 13, 022109 (2006).

${ }^{3}$ W. D. White, J. H. Malmberg, and C. F. Driscoll, Phys. Rev. Lett. 49, 1822 (1982).

${ }^{4}$ G. Bettega, F. Cavaliere, B. Paroli, M. Cavenago, R. Pozzoli, and M.
Romé, Phys. Plasmas 14, 102103 (2007).

${ }^{5}$ A. C. Cass, "Experiments on vortex symmetrization in magnetized electron columns," Ph.D. dissertation, University of California at San Diego (1998).

${ }^{6}$ N. S. Pillai and R. W. Gould, Phys. Rev. Lett. 73, 2849 (1994).

${ }^{7}$ R. J. Briggs, J. D. Daugherty, and R. H. Levy, Phys. Fluids 13, 421 (1970).

${ }^{8}$ M. Amoretti, G. Bettega, F. Cavaliere, M. Cavenago, F. De Luca, R. Pozzoli, and M. Romé, Rev. Sci. Instrum. 74, 3991 (2003).

${ }^{9}$ J. Millman, Microelectronics (McGraw-Hill, New York, 1979), p. 573.

${ }^{10}$ G. Bettega, F. Cavaliere, M. Cavenago, F. De Luca, A. Illiberi, R. Pozzoli, and M. Romé, Phys. Plasmas 13, 112102 (2006).

${ }^{11}$ R. C. Davidson, An Introduction to the Physics of Nonneutral Plasmas (Addison-Wesley, Redwood City, 1990), p. 304.

${ }^{12}$ S. A. Prasad and T. M. O’Neil, Phys. Fluids 26, 665 (1983).

${ }^{13}$ S. N. Rasband and R. L. Spencer, Phys. Plasmas 10, 948 (2003), and references therein. 